\title{
'Proof of EHS beyond all reasonable doubt'. Comment on: Leszczynski D. Review of the scientific evidence on the individual sensitivity to electromagnetic fields (EHS). Rev Environ Health 2021; https://doi.org/10.1515/reveh-2021-0038. Online ahead of print
}

https://doi.org/10.1515/reveh-2021-0101

Received July 13, 2021; accepted July 20, 2021;

published online August 2, 2021

Keywords: electromagnetic hypersensitivity; environmental intolerance; scientific proof beyond all doubt.

\section{Proof of EHS beyond all reasonable doubt}

Leszczynski's review [1] included two important conclusions. Firstly, the need for the WHO, ICNIRP, ICES and governmental organisations to revise their denial of the link between EHS and electromagnetic fields (EMFs) because the data is of insufficient quality for proof of the lack of causality. Secondly, instead of studying a nocebo effect, research should focus on finding "suitable biochemical and biophysical markers" for symptoms in each EHS individual.

However, the review also stated that "So far, scientists were unable to find causality link between symptoms experienced by sensitive persons and the exposures to EMF”. This comprehensive assertion does not seem to reflect all the scientific evidence.

The criteria for proof, here onwards defined as beyond all reasonable doubt, differ between causality for an environmental intolerance (EI), such as EHS, and causality for a bacterial or viral disease. For the latter, there is usually a cellular organism or virion. For an EI, there can be

*Corresponding author: Michael Bevington, Chair of Trustees, Electrosensitivity UK, London, UK, http://www.es-uk.info/, E-mail:michael@es-uk.info several triggers and pathways affecting many organs, tissues and cells. EI can also be caused by genetics and viruses.

Proof of causality for an EI necessarily depends, as for any cause, on sequential temporality. This temporal sequence is usually evident in a repeatable physiological symptom(s) or change(s) often measurable by an objective marker(s). However, each individual may react differently to a given environmental stimulus. Scientific proof of health causality usually also requires a known mechanism. In the case of an electromagnetic EI such as sunburn or skin cancer from sunshine, individual differences have long been known, while a mechanism in the form of a genetic defect in DNA repair was discovered in 1968.

For EHS, another electromagnetic EI, differences in individuals' symptoms from man-made EMFs have been known since 1733. In 2008 the first genetic variant associated with EMF sensitivity was discovered, the XRCC1 Ex9+16A allele, a DNA repair polymorphism, linked with childhood leukaemia near substations and powerlines [2]. In 2014 it was reported that people with EHS were 9.7 times more likely to have GSTM1 + GSTT1 null genotypes [3], indicating a susceptibility to oxidative stress. This genetic variation can also increase the risk of multiple sclerosis, some cancers, Alzheimer's and asthma, each sometimes associated with EHS. Such genetic variants seem more common at higher than lower latitudes and in women than men, with others associated with higher levels of mercury. EHS symptoms are also associated with some demyelinating neurodegenerative conditions.

A causal link between electrosensitive symptoms and EMF exposures has also been proved for other mechanistic pathways in addition to genetic. Calcium flux through membrane depolarisation was discovered in 
1974, involving the radical pair mechanism at ELF up to $\mathrm{MHz}$, as in modulated cell phone signals. Unmodulated $\mathrm{GHz}$ radiofrequency can generate oxidative stress and may act through ferritin, calcium spikes or water modification, but further proof is needed. Other pathways include cryptochromes [4]. Such EMF sensitivity occurs in $100 \%$ of people subliminally, and in $30 \%$ consciously [5]. Hypersensitivity is associated with the $1.2 \%$ severely disabled by EMFs.

Scientific proof also partly depends on repeatability, as in provocation tests, either subliminal or conscious. Such tests were first applied to EHS in the 1980s by Dr Cyril Smith, who originated the term 'Electromagnetic Hypersensitivity', and Dr Jean Munro. Following near-quantum and non-linear insights by Professor Herbert Frölich, they first identified the specific frequencies to which an individual was sensitive. They then reproduced the EMF exposure, proving that positive provocation tests of screened subjects could be repeated accurately. Similar tests were used in 1991 at the Environmental Health Center, Texas, by Dr William Rea, who held the world's first professorship in environmental medicine at the University of Surrey in 1988. These achieved $100 \%$ success by screening for specific frequencies and rejecting $84 \%$ of subjects without consistent responses [6]. Dr Magda Havas and Professor Andrew Marino confirmed this through similar diagnostic protocols. High accuracy in blinded provocation tests was also recorded for individuals in studies without screening, as at Essex University in 2007, but their individual data were not published and therefore lost in averaging. Some unscreened studies hypothesised without evidence a different condition, namely a nocebo effect or electrophobia, known since 1903, but inapplicable to unaware adults, some of whom suffer physiological EHS.

Further proof of EMF causality for EHS symptoms includes the $20 \%$ of subjects known since 1998 to suffer electrosensitivity symptoms during Transcranial Magnetic Stimulation. Likewise, walking fast through magnetic fields near MRI scanners can induce electric currents causing specific EHS symptoms, with a small hypersensitive subset. Similarly, some people are sensitive to geomagnetic disturbances and thunderstorms [7].

Clinical evidence also contributes to proof of EHS. Specific EHS symptoms were identified from 1932 in Eastern Europe and the USSR, usually among people occupationally exposed, such as radar, radio or electricity workers. As EHS spread into the general population with the use of cell phones, Wi-Fi and smart metres, specialist EHS centres assessed greater numbers, such as Professor
Dominique Belpomme's in Paris. In 2015 he published the first comprehensive study of objective molecular biomarkers including cerebral blood perfusion scans, showing that EHS is a multi-systemic EI like chemical sensitivity. In 2021 Belpomme led 32 international experts requesting that the WHO acknowledges EHS as a distinct neuropathological disorder and includes it in its International Classification of Diseases [8]. In 2017 Dr Gunnar Heuser published evidence from fMRI scans of brain effects [9]. Similar scans helped convince a 2020 government report that the U.S. diplomats in Cuba were harmed by radiofrequency weapons.

In the 1930s, sufficient proof that adverse health symptoms were caused by non-thermal EMF exposure led to the first radiofrequency guidelines being non-thermal. Non-thermal effects of radiofrequency were shown as primary, with heating secondary. In 1953 sensitivity symptoms were shown to include cancers among radar workers and, from 2004, among people living nearer a cell phone tower compared with those further away, while in 1979 increased leukaemia was found among people living near powerlines. The IARC recognised non-thermal effects by classifying radiofrequency EMFs from cell phones as a 2B carcinogen in 2011. This led to courts from 2012 fining employers, and compensating EHS employees severely affected by non-thermal EMFs.

The scientific proof of the causal link between symptoms and EMF exposures has also been accepted since the 1990s by insurers. They refuse to underwrite EMF risks except as high category like asbestos, another carcinogen. Following Sweden in 2000, like the WHO in 2005, some countries specifically recognise EHS as functionally disabling and requiring accommodation under equality legislation. In 2020 a Dutch appeal judge recognised a person with EHS as an interested party in siting a cell phone tower.

Finally, two of the review's three "essential, but still unanswered" questions - the EMF levels tolerated without conscious adverse effects and the counter-measures to protect people with EHS - were answered in some respects by the EUROPAEM EMF Guideline 2016 [10], subsequently adapted for the International Guidelines on Non-Ionising Radiation of 2018. Typically, public health levels to prevent harm are set 10 to 50 times below the lowest experimentally proven health effects to accommodate exceptionally hypersensitive individuals. However, some non-thermal guidelines include the duration of EMF exposure to facilitate greater flexibility, while also protecting sleep locations and those proven as the most sensitive groups in society. 
Research funding: None declared.

Author contributions: Author has accepted responsibility for the entire content of this manuscript and approved its submission.

Competing interests: Author states no conflict of interest. Informed consent: Not applicable.

Ethical approval: The comments expressed here do not involve new research on humans or animals.

\section{References}

1. Leszczynski D. Review of the scientific evidence on the individual sensitivity to electromagnetic fields (EHS). Rev Environ Health 2021 Jul 6. https://doi.org/10.1515/reveh-2021-0038 [Epub ahead of print].

2. Yang Y, Jin X, Yan C, Tian Y, Tang J, Shen X. Case-only study of interactions between DNA repair genes ( $\mathrm{hMLH1}$, APEX1, MGMT, XRCC1 and XPD) and low-frequency electromagnetic fields in childhood acute leukemia. Leuk Lymphoma 2008; 49:2344-50.

3. De Luca C, Thai JC, Raskovic D, Cesareo E, Caccamo D, Trukhanov A, et al. Metabolic and genetic screening of electromagnetic hypersensitive subjects as a feasible tool for diagnostics and intervention. Mediat Inflamm 2014;2014:924184.

4. Sherrard RM, Morellini N, Jourdan N, El-Esawi M, Arthaut L-D, Niessner $C$, et al. Low-intensity electromagnetic fields induce human cryptochrome to modulate intracellular reactive oxygen species. PLoS Biol 2018;16:e2006229.

5. Bevington $M$. The prevalence of people with restricted access to work in manmade electromagnetic environments. J Environ Health Sci 2019;5:1-12.

6. Rea WJ, Pan Y, Fenyves EJ, Sujisawa I, Suyama H, Samadi N, et al. Electromagnetic field sensitivity. J Bioelectr 1991;10:241-56.

7. Panagopoulos DJ, Balmori A. On the biophysical mechanism of sensing atmospheric discharges by living organisms. Sci Total Environ 2017;599-600:2026-34.

8. Belpomme D, Carlo GL, Irigaray P, Carpenter DO, Hardell L, Kundi $M$, et al. The critical importance of molecular biomarkers and imaging in the study of electrohypersensitivity. A Scientific Consensus International Report. Int J Mol Sci 2021;22:7321.

9. Heuser G, Heuser SA. Functional brain MRI in patients complaining of electrohypersensitivity after long term exposure to electromagnetic fields. Rev Environ Health 2017;32:291-9.

10. Belyaev I, Dean A, Eger H, Hubmann G, Jandrisovits R, Kern M, et al. EUROPAEM EMF Guideline 2016 for the prevention, diagnosis and treatment of EMF-related health problems and illnesses. Rev Environ Health 2016;31:363-97. 